\title{
On tame embeddings of solenoids into 3-space
}

September 26, 2018

BOJU JIANG, jiangbj@math.pku.edu.cn

Depart. of Mathematics, Peking University, Beijing 100871, China

SHICHENG WANG, wangsc@math.pku.edu.cn

Depart. of Mathematics, Peking University, Beijing 100871, China

HAO ZHENG, zhenghao@mail.sysu.edu.cn

Depart. of Mathematics, Zhongshan University, Guangzhou 510275, China

QING ZHOU, qingzhou@sjtu.edu.cn

Depart. of Mathematics, Jiaotong University, Shanghai 200030, China

\begin{abstract}
Solenoids are "inverse limits" of the circle, and the classical knot theory is the theory of tame embeddings of the circle into the 3 -space. We give some general study, including certain classification results, of tame embeddings of solenoids into the 3 -space as the "inverse limits" of the tame embeddings of the circle.

Some applications are discussed. In particular, there are "tamely" embedded solenoids $\Sigma \subset \mathbb{R}^{3}$ which are strictly achiral. Since solenoids are non-planar, this contrasts sharply with the known fact that if there is a strictly achiral embedding $Y \subset \mathbb{R}^{3}$ of a compact polyhedron $Y$, then $Y$ must be planar.
\end{abstract}

\section{Introduction and motivations}

The classical knot theory is the theory of tame embeddings of the circle into the 3space, which has become a central topic in mathematics. The classical theory of knots has many generalizations and variations: from the circle to graphs, from the circle to higher dimensional spheres, from tame embeddings to wild embeddings, and so no. 
In the present note, we try to setup a beginning of another generalization: the tame embeddings of solenoids into the 3-space. In such a study topology and dynamics interact well.

The solenoids may be first defined in topology by Vietoris in 1927 for 2-adic case [V] and by many others later for general cases, and introduced into dynamics by Smale in 1967 [S]. Solenoids can be presented either in a rather geometric way (intersections of nested solid tori, see Definition 2.2) or in a rather algebraic way (inverse limits of self-coverings of the circle, see Definition 2.1), or in rather dynamics way (mapping tori over the Cantor set, see $[\mathrm{Mc}])$.

The precise definition of tame embedding of solenoids into the 3 -space $\mathbb{R}^{3}$ will be given in $\S 2$, but the intuition is quite naive. Recall we identify $S^{1}$ with the centerline of the solid tours $S^{1} \times D^{2}$, and say an embedding $S^{1} \subset \mathbb{R}^{3}$ is tame if the embedding can be extended to an embedding $S^{1} \times D^{2} \subset \mathbb{R}^{3}$. Similarly we consider a solenoid $\Sigma$ as the nested intersections of solid tori (the defining sequence of $\Sigma$ ), and say an embedding $\Sigma \subset \mathbb{R}^{3}$ is tame if the embedding can be extended to an embedding of those solid tori into $\mathbb{R}^{3}$.

Solenoids themselves usually are considered as "wild" set. What motivated us originally to study the tame embeddings of solenoids was trying to find a non-planar set which admits a strictly achiral embedding into the 3 -space.

An embedding $A \subset \mathbb{R}^{3}$ is called strictly achiral, if $A$ stays in the fixed point set of an orientation reversing homeomorphism $r: \mathbb{R}^{3} \rightarrow \mathbb{R}^{3}$. Obviously any planar set has a strictly achiral embedding. Indeed there is a simple relation between the two notions of achirality and planarity for compact polyhedra: If there is a strictly achiral embedding $Y \subset \mathbb{R}^{3}$ of a compact polyhedron $Y$, then $Y$ must be planar [JW]. In the sequel, it is natural to ask whether the relation from [JW] still holds when compact polyhedra are replaced by continua (i.e. compact, connected metric spaces).

The solenoids are promising, and indeed are proved to be in $\S 4$, counterexamples to the question, because on the one hand they are continua realized as inverse limits of planar sets, on the other hand they are non-planar themselves (see [Bin], and [JWZ] for a short proof). In order to design a strictly achiral embedding $\Sigma \subset \mathbb{R}^{3}$ for a solenoid $\Sigma$, we need careful and deep discussions about the tame embeddings of solenoids.

Among other motivations, when a solenoid $\Sigma \subset S^{3}$ is realized as a hyperbolic attractor of a dynamics (called Smale solenoid), the embedding $\Sigma \subset S^{3}$ is automatically tame. Our study also gives some application on this aspect.

The contents of the paper are as follows.

In $\S 2$, we give the precise definitions of tame solenoids and related notions. We present a lemma about convergence of homeomorphisms which will be repeatedly used in the paper. With this lemma we give an alternative description of the tame solenoids via the language of mapping torus. For comparison we also construct examples of non- 
tame embeddings of solenoids vis the mapping torus description.

In $\S 3$, we classify the tame solenoids in the 3 -sphere. The classification is based on an important notion, the "maximal" defining sequences of tame solenoids. We also give applications including (1) the knotting, linking and invariants of tame solenoids;

(2) there are uncountably many unknotted 2-adic tame solenoids; (3) the number of Smale solenoids $\Sigma \subset S^{3}$ realizing the periodic solenoids of type $\left(w_{1}, \ldots, w_{k}\right)$ is finite if all $w_{n} \leq 3$, and is countably infinite otherwise.

In $\S 4$, we give criterions of when a tame solenoid in the 3 -sphere is achiral or strictly achiral in term of its defining sequence, and construct strictly achiral tame solenoids to fulfil our original motivation. Indeed we give a simple criterion when a solenoid has a strictly achiral embedding into the 3-sphere.

All terminologies not defined are standard. For 3-manifolds, see [Ja]; for knot theory see $[\mathrm{A}]$; and for braid theory, see [Bir].

\section{Tame embeddings of solenoids, Preliminaries}

\subsection{Definitions of solenoids and their tame embeddings}

Let $N=D^{2} \times S^{1}$ be the solid torus, where $D^{2}$ is the unit disc and $S^{1}$ is the unit circle. Then $N$ admits a standard metric. A meridian disk of $N$ is a $D^{2}$ slice of $N$. A framing of $N$ is a circle on $\partial N$ which meets each meridian disk of $N$ at exactly one point.

Definition 2.1. (1) For a sequence of maps $\left\{\phi_{n}: X_{n} \rightarrow X_{n-1}\right\}_{n \geq 1}$ between continua, the inverse limit is defined to be the subspace

$$
\Sigma=\left\{\left(x_{0}, x_{1}, \ldots, x_{n}, \ldots\right) \mid x_{n} \in X_{n}, x_{n-1}=\phi_{n}\left(x_{n}\right)\right\}
$$

of the product space $\prod_{n=0}^{\infty} X_{n}$.

(2) The inverse limit of a sequence of covering maps $\left\{\phi_{n}: S^{1} \rightarrow S^{1}\right\}_{n \geq 1}$, where $\phi_{n}$ is of degrees $w_{n} \neq 0$, is called a solenoid of type $\varpi=\left(w_{1}, w_{2}, \ldots, w_{n}, \ldots\right)$.

Definition 2.2. (1) Call an embedding $e: N \rightarrow \operatorname{int} N$, or simply call the image $e(N)$, a thick braid of winding number $w$ if $e$ preserves the $D^{2}$-fiberation and descends to a covering map $S^{1} \rightarrow S^{1}$ given by $e^{i t} \mapsto e^{i w t}$. Note that the composition of finitely many thick braids is also a thick braid.

(2) Let $\left\{e_{n}: N \rightarrow N\right\}_{n \geq 1}$ be an infinite sequence of thick braids of winding numbers $w_{n} \neq 0$. Let $\psi_{n}=e_{n} \circ \cdots \circ e_{2} \circ e_{1}$ and $N_{n}=\psi_{n}(N)$. Then we have an infinite sequence $N=N_{0} \supset N_{1} \supset N_{2} \supset \cdots \supset N_{n} \supset \cdots$ of thick braids. If the diameters of the meridian disks of $N_{n}$ tend to zero uniformly as $n \rightarrow \infty$ then we call $\Sigma=\cap_{n \geq 0} \psi_{n}(N)=\cap_{n>0} N_{n}$ a solenoid of type $\varpi=\left(w_{1}, w_{2}, \ldots, w_{n}, \ldots\right)$. 
There is quite a rich theory about solenoids developed in 1960-1990's. We just list some basic facts (see $[\mathrm{Mc}],[\mathrm{R}]$ and references therein) as

Theorem 2.3. (1) The above two definitions of solenoids are equivalent; each solenoid is determined by its type $\varpi$.

(2) Two solenoids $\Sigma$ and $\Sigma^{\prime}$ of types $\varpi$ and $\varpi^{\prime}$ respectively are homeomorphic if deleting finitely many terms from $\varpi$ and $\varpi^{\prime}$ can make them identical. Moreover, $\Sigma$ is the circle if and only if all except finitely many $w_{n}$ are equal to \pm 1 .

(3) Each solenoid $\Sigma$ is connected, compact and has topological dimension one. Moreover, if $\Sigma$ is not the circle, then $\Sigma$ has uncountably many path components.

We assume below that all winding numbers involved in the definition of solenoid are greater than 1, unless otherwise specified. In particular, a solenoid is not the circle.

Definition 2.4. (1) In Definition 2.2, call $\left\{N_{n}\right\}_{n \geq 0}$ a defining sequence of the solenoid $\Sigma$, and call $\Sigma \subset N$ a standard embedding of $\Sigma$ in the solid torus $N$.

(2) $\Sigma \subset N$ is called a tame embedding of $\Sigma$ in the solid torus $N$, if there is a homeomorphism $f:(N, \Sigma) \rightarrow\left(N, \Sigma^{\prime}\right)$ for some standard embedding $\Sigma^{\prime} \subset N$; then call $\left\{f^{-1}\left(N_{n}^{\prime}\right)\right\}_{n \geq 0}$ a defining sequence of $\Sigma$, where $\left\{N_{n}^{\prime}\right\}_{n>0}$ is a defining sequence of $\Sigma^{\prime}$.

(3) An embedding $\Sigma \subset S^{3}$ of solenoid is called tame, if the embedding can be factored as $\Sigma \subset N \subset S^{3}$ in which $\Sigma \subset N$ is tame; then each defining sequence $\left\{N_{n}\right\}_{n \geq 0}$ of $\Sigma \subset N$ is also considered as a defining sequence of $\Sigma \subset S^{3}$, and we have $S^{3} \supset N=N_{0} \supset N_{1} \supset N_{2} \supset \cdots \supset N_{n} \supset \cdots \supset \Sigma$.

Remark 2.5. From Definition 2.4, for each defining sequence $\left\{N_{n}\right\}_{n \geq 0}$ of a tame solenoids in $S^{3}$, we always assume that the $D^{2}$-slices of all $N_{i}$ are coherent.

Definition 2.6. Call two tame solenoids $\Sigma, \Sigma^{\prime} \subset S^{3}$ equivalent if there is an orientation preserving homeomorphism $f: S^{3} \rightarrow S^{3}$ such that $f(\Sigma)=\Sigma^{\prime}$.

Definition 2.7. Say two defining sequences $\left\{N_{n}\right\}_{n \geq 0}$ and $\left\{N_{n}^{\prime}\right\}_{n \geq 0}$ of tame solenoids in $S^{3}$ are strongly equivalent, if there are orientation preserving homeomorphism $f_{0}$ : $\left(S^{3}, N_{0}\right) \rightarrow\left(S^{3}, N_{0}^{\prime}\right)$ and orientation preserving homeomorphisms $f_{n}:\left(N_{n-1}, N_{n}\right) \rightarrow$ $\left(N_{n-1}^{\prime}, N_{n}^{\prime}\right)$ with $\left.f_{n}\right|_{\partial N_{n-1}}=\left.f_{n-1}\right|_{\partial N_{n-1}}$ for $n \geq 1$. Say $\left\{N_{n}\right\}_{n \geq 0}$ and $\left\{N_{n}^{\prime}\right\}_{n \geq 0}$ are equivalent, if $\left\{N_{k+n}\right\}_{n \geq 0}$ and $\left\{N_{k^{\prime}+n}^{\prime}\right\}_{n \geq 0}$ are strongly equivalent for some non-negative integers $k$ and $k^{\prime}$.

Remark 2.8. (1) A defining sequence $\left\{N_{n}\right\}_{n \geq 0}$ of a tame solenoid $\Sigma \subset S^{3}$ carries the information of the braiding of $N_{n}$ in $N_{n-1}$ and the knotting of $N_{n}$ in $S^{3}$. The winding numbers $w_{n}$, the simplest invariant of the braiding of $N_{n}$ in $N_{n-1}$, give rise to the type of the abstract solenoid $\Sigma$. 
(2) Suppose $\Sigma \subset S^{3}$ is a tame embedding given by a defining sequence $\left\{N_{n}\right\}_{n \geq 0}$, then any infinite subsequence of $\left\{N_{n}\right\}_{n \geq 0}$ is a defining sequence of the same embedding.

(3) Definitions 2.4 2.7 also apply to other 3-manifolds in the obvious way.

In this paper we view each braid $\beta$ is defined in $D^{2} \times[0,1]$ with ends stay in $D^{2} \times\{0,1\}$. The closure $\bar{\beta} \subset D^{2} \times S^{1}$ is obtained by identifying $D^{2} \times 0$ and $D^{2} \times 1$ via the identity. Conversely, for each closed braid $\bar{\beta} \subset D^{2} \times S^{1}$, cutting $D^{2} \times S^{1}$ open along a $D^{2}$ slice yields a braid $\beta \subset D^{2} \times[0,1]$ up to conjugacy. Therefore, we have the 1-1 correspondence between the set of closed braids of winding number $n$ and the set of conjugacy classes of braids on $n$ strands.

Note that a tubular neighborhood of a closed braid in $D^{2} \times S^{1}$ is a thick braid and, conversely, a framing of a thick braid in $D^{2} \times S^{1}$ is a closed braid.

\subsection{Convergence of homeomorphisms}

The following lemma will be used repeatedly in this paper.

Lemma 2.9. Let $X, Y$ be compact metric spaces and $\left\{f_{n}: X \rightarrow Y\right\}_{n \geq 0}$ be a series of homeomorphisms. If there exist subsets $\left\{U_{n}\right\}_{n \geq 0}$ of $X$ and positive numbers $\left\{\varepsilon_{n}\right\}_{n \geq 0}$ such that

(1) $U_{n} \subset U_{n-1}$ and $\left.f_{n}\right|_{X \backslash U_{n-1}}=\left.f_{n-1}\right|_{X \backslash U_{n-1}}$,

(2) $\lim _{n \rightarrow \infty} \varepsilon_{n}=0$ and $d\left(f_{m}(x), f_{n}(x)\right) \leq \varepsilon_{n}, d\left(f_{m}^{-1}(y), f_{n}^{-1}(y)\right) \leq \varepsilon_{n}$ for $x \in X$, $y \in Y, m \geq n$,

(3) Both $\cap_{n \geq 0} U_{n}$ and $\cap_{n \geq 0} f\left(U_{n}\right)$ have no interior points, then $f_{n}$ uniformly converges to a homeomorphism $f:\left(X, \cap_{n \geq 0} U_{n}\right) \rightarrow\left(Y, \cap_{n \geq 0} f_{n}\left(U_{n}\right)\right)$. Moreover, if in addition,

(4) $X=Y$ and $d\left(f_{n}(x), x\right) \leq \varepsilon_{n}$ for $x \in U_{n}$, then $\cap_{n \geq 0} U_{n}$ lies in the fixed point set of $f$.

Proof. Since both $X$ and $Y$ are compact, it follows from Condition (2) that $f_{n}$ and $f_{n}^{-1}$ uniformly converge to continuous maps $f: X \rightarrow Y$ and $g: Y \rightarrow X$, respectively. By Condition (1), we have $\left.f\right|_{X \backslash U_{n}}=\left.f_{n}\right|_{X \backslash U_{n}}$ and $\left.g\right|_{X \backslash f\left(U_{n}\right)}=\left.f_{n}^{-1}\right|_{X \backslash f\left(U_{n}\right)}$ hence $\left.f\right|_{X \backslash \cap_{n \geq 0} U_{n}}$ is the inverse of $\left.g\right|_{Y \backslash \cap_{n \geq 0} f_{n}\left(U_{n}\right)}$.

Since $X \backslash \cap_{n \geq 0} U_{n}$ is dense in $X$ by Condition (3), on which the continuous map $g f: X \rightarrow X$ acts as the identity, it follows that $g f=\operatorname{id}_{X}$. Similarly, we have $f g=\mathrm{id}_{Y}$. So the conclusion follows.

The "Moreover" part is clear.

\subsection{Tame solenoids as mapping tori}

Definition 2.10. Let $C \subset D^{2}$ be a Cantor set. Say an orientation preserving homeomorphism $f:\left(D^{2}, C\right) \rightarrow\left(D^{2}, C\right)$ factors through nested disks if there exist subsets 
$\left\{U_{n}\right\}_{n \geq 0}$ of $D^{2}$ such that $U_{n+1} \subset$ int $U_{n}, \cap_{n \geq 0} U_{n}=C$ and each $U_{n}$ is a disjoint union of 2-disks on which $f$ cyclically permutes the components of $U_{n}$.

Theorem 2.11. A solenoid $\Sigma \subset S^{3}$ is tame if and only if $\Sigma$ has a neighborhood $N$ such that $(N, \Sigma)$ is homeomorphic to the mapping torus of a homeomorphism $f$ : $\left(D^{2}, C\right) \rightarrow\left(D^{2}, C\right)$ which factors through nested disks.

The Proof depends on the following lemma which will be also used in several other places of the paper.

Proof of Theorem 2.11. Sufficiency is clear: Suppose $f:\left(D^{2}, C\right) \rightarrow\left(D^{2}, C\right)$ is an orientation preserving homeomorphism which factors through nested disks $\left\{U_{n}\right\}_{n \geq 0}$. Then for each $n \geq 0$, let $N_{n}=U_{n} \times[0,1] / f$. Then $N_{n+1}$ is a thick braid in $N_{n}$ and $\cap_{n \geq 0} N_{n}=C \times[0,1] / f \subset N_{0}$ is a tame embedding of solenoid with defining sequence $\left\{N_{n}\right\}_{n \geq 0}$.

Necessity. Let $\Sigma \subset N$ be a tame embedding with defining sequence $\left\{N_{n}\right\}_{n \geq 0}$. We will show that $(N, \Sigma)$ is homeomorphic to the mapping torus of a homeomorphism $f:\left(D^{2}, C\right) \rightarrow\left(D^{2}, C\right)$ which factors through nested disks. Fix a meridian disk $D$ of $N$. For $n \geq 0$, let $U_{n}=D \cap N_{n}$. Clearly $U_{n}$ are disjoint disks, $U_{n+1} \subset \operatorname{int} U_{n}$, and $C=\cap_{n \geq 0} U_{n}$ is a Cantor set in $D^{2}$.

Now recursively define homeomorphism $f_{n}:\left(D, U_{n}\right) \rightarrow\left(D, U_{n}\right)$ such that $\left.f_{n}\right|_{D \backslash U_{n-1}}=$ $\left.f_{n-1}\right|_{D \backslash U_{n-1}}$ and $T_{f_{n}} \cong\left(N_{0}, N_{n}\right)$, where $T_{f_{n}}$ is the mapping torus of $f_{n}$. Since $N_{n}$ is connected, $f_{n}$ permutes the components of $U_{n}$ cyclically. Then recursively define $D^{2}$ fiberation preserving homeomorphism $\tilde{f}_{n}: T_{f_{n}} \rightarrow\left(N_{0}, N_{n}\right)$ such that $\tilde{f}_{n}$ is identical to $\tilde{f}_{n-1}$ on the mapping torus over $D \backslash U_{n-1}$. Since the diameters of the components of $U_{n}$ and the meridian disks of $N_{n}$ tend to zero uniformly as $n \rightarrow \infty$ and $\tilde{f}_{n}$ is $D^{2}$-fiberation preserving, by Lemma $2.9 f_{n}$ uniformly converges to a homeomorphism $f:(D, D \cap \Sigma) \rightarrow(D, D \cap \Sigma)$ and $\tilde{f}_{n}$ uniformly converges to a homeomorphism $\tilde{f}: T_{f} \rightarrow\left(N_{0}, \Sigma\right)$. It is clear that $f$ factors through $\left\{U_{n}\right\}_{n \geq 0}$.

\subsection{Non-tame embeddings of solenoids}

As there are non-tame embeddings of the circle into the 3-space, there are non-tame embeddings of solenoids. In fact, any tame embedding of solenoid can be modified in a simple way to a non-tame embedding, which we illustrate below by a concrete example.

Suppose nested disks $\left\{U_{n}\right\}_{n \geq 0}$ and an orientation preserving homeomorphism $f$ : $\left(D^{2}, C\right) \rightarrow\left(D^{2}, C\right)$ which factors through $\left\{U_{n}\right\}_{n \geq 0}$ have been chosen as in previous subsection so that each $U_{n}$ consists of $2^{n}$ disks. Label those disks by $U_{1}=D_{0} \cup D_{1}$, $U_{2}=D_{00} \cup D_{01} \cup D_{10} \cup D_{11}$ with $D_{i j} \subset D_{i}$, where $i, j \in\{0,1\}$, and so on. For simplicity, denote by $D_{n}^{\prime}, D_{n}^{\prime \prime}$ the components $D_{11 \ldots 10}, D_{11 \ldots 11} \subset U_{n}$ respectively. 


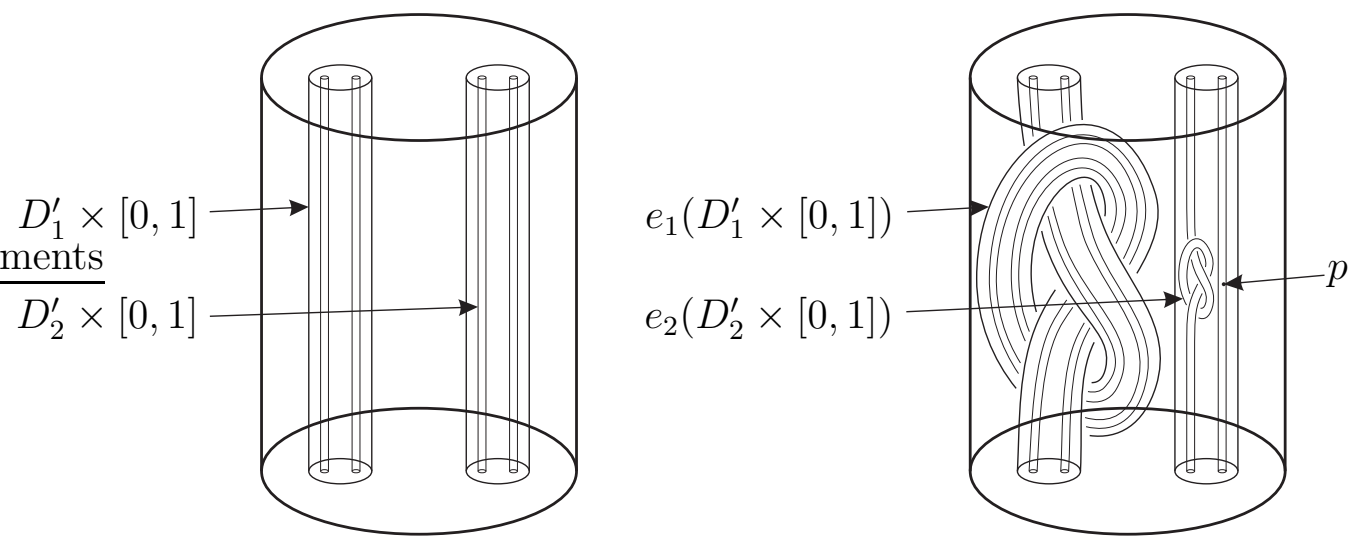

Figure 1

In the left side of Figure 1, we see the embedding sequence $D^{2} \times[0,1] \supset U_{1} \times$ $[0,1] \supset U_{2} \times[0,1] \supset \cdots \supset C \times[0,1]$. We define a re-embedding $e: C \times[0,1] \subset$ $D^{2} \times[0,1]$ relative to $D^{2} \times\{0,1\}$ by assembling the re-embeddings $\left\{e_{n}: D_{n}^{\prime} \times[0,1] \subset\right.$ $\left.D^{2} \times[0,1]\right\}_{n \geq 1}$, where $e_{n}$ is constructed by replacing an unknotted middle portion of $D_{n}^{\prime} \times[0,1]$ by a knotted one such that the knotted portion of $D_{n}^{\prime} \times[0,1]$ is contained in a 3 -ball $B_{n} \subset\left(D_{n-1}^{\prime \prime} \backslash D_{n}^{\prime}\right) \times[0,1]$, the diameter of $B_{n}$ tending to zero and $B_{n}$ converging to a point $p$. The situation is indicated as the right side of Figure 1 . Note that for each small open 3-ball neighborhood $B$ of $p$ there is an arc component of $B \cap e(C \times[0,1])$ which is knotted in $B$. Denote this property by $(*)$.

Let $T_{f}$ be the mapping torus of the homeomorphism $f:\left(D^{2}, C\right) \rightarrow\left(D^{2}, C\right)$. Then the quotient of $e(C \times[0,1]) \subset D^{2} \times[0,1]$ in $T_{f}$ is an embedding of 2-adic solenoid into the solid torus $T_{f}$. This embedding is not tame, since a tame embedding do not have property $(*)$.

\section{Classification and applications}

\subsection{Maximal defining sequences}

As can be easily seen, a tame solenoid $\Sigma \subset N$ has a lot of defining sequences and there is no way to choose a "minimal" one from them. However, "maximal" defining sequence of a tame solenoid can be defined in the sense that every other defining sequence is equivalent to a subsequence of it (see Proposition 3.2).

Before giving the precise definition we fix a notation. For a properly embedded surface $S$ (resp. an embedded 3-manifold $P$ ) in a 3-manifold $M$, we use $M \backslash S$ (resp. $M \backslash P$ ) to denote the resulting manifold obtained by splitting $M$ along $S$ (resp. removing the interior of $P$ ).

Definition 3.1. Call a defining sequence $\left\{N_{n}\right\}_{n \geq 0}$ of a tame solenoid $\Sigma \subset S^{3}$ maximal if $N_{n} \backslash N_{n+1}$ contains no essential torus for each $n \geq 0$. 
Proposition 3.2. Every tame solenoid $\Sigma \subset S^{3}$ has a maximal defining sequence. Moreover, if $\left\{N_{n}\right\}_{n \geq 0}$ is a maximal defining sequence of $\Sigma$ then every defining sequence of $\Sigma$ is equivalent to a subsequence of $\left\{N_{n}\right\}_{n \geq 0}$. Hence all maximal defining sequences of $\Sigma$ are equivalent.

The following lemma will be repeatedly used in this paper.

Lemma 3.3. Suppose $N^{\prime}$ is a thick braid in $N$ and $\Gamma$ is the JSJ-decomposition tori of $N \backslash N^{\prime}$. Then

(1) each component $T$ of $\Gamma$ bounds a solid torus $N^{*}$ such that $N^{*}$ is a thick braid in $N$ and $N^{\prime}$ is a thick braid in $N^{*}$;

(2) each component of $\left(N \backslash N^{\prime}\right) \backslash \Gamma$ contains no essential torus;

(3) for each solid torus $N^{\prime \prime}$ with $N^{\prime} \subset N^{\prime \prime} \subset N$, $\partial N^{\prime \prime}$ is isotopic in $N \backslash N^{\prime}$ to a component of $\Gamma \cup \partial N \cup \partial N^{\prime}$.

Proof. (1) Let $w$ be the winding number of $N^{\prime}$ in $N$ and let $D$ be a meridian disk of $N$ which meets $N^{\prime}$ at $w$ meridian disks of $N^{\prime}$. Then $\left(N \backslash N^{\prime}\right) \backslash D \cong P_{w} \times I$, where $P_{w}$ is the $w$-punctured disc. Isotope $T$ so that $T \cap D$ has minimum number of components. Then a standard argument in 3-manifold topology shows that each component of $T \backslash D$ in $P_{w} \times I$ is a vertical annulus which separates a vertical $D^{2} \times I$ from $N \backslash D$. Therefore $T$ bounds a solid torus $N^{*}$ which is a thick braid in $N$ and clearly $N^{\prime}$ is a thick braid in $N^{*}$.

(2) Let $Q$ be a component of $\left(N \backslash N^{\prime}\right) \backslash \Gamma$. By JSJ theory [Ja], $Q$ is either simple hence contains no essential torus by definition, or a Seifert piece. Suppose $Q$ is a Seifert piece. Then $Q$ is also a Seifert piece of knot complement with incompressible boundary. According to [Ja, IX.22. Lemma], $Q$ is either a torus knot space, or a $P_{w} \times S^{1}$ where $P_{w}$ is the $w$-punctured disc with $w \geq 2$, or a cable space. Since $\partial Q$ has at least two components, $Q$ is not the torus knot space. By the conclusion of (1) and the fact that $N^{\prime}$ is connected, one can verify that there is no embedding of $P_{w} \times S^{1}$ in $N \backslash N^{\prime}$ with incompressible boundary for $w \geq 2$. Therefore $Q$ is a cable space. It is known that a cable space contains no essential torus.

(3) By the conclusions of (2), it suffices to show that $\partial N^{\prime \prime}$ is incompressible in $N \backslash N^{\prime}$. Suppose $\partial N^{\prime \prime}$ has a compressing disk $D$ in $N \backslash N^{\prime}$. Then after surgery on $D$ we will get a separating 2-sphere $S^{2}$ in $N \backslash N^{\prime}$ such that each component of $\left(N \backslash N^{\prime}\right) \backslash S^{2}$ contains a boundary torus, which contradicts that $N \backslash N^{\prime}$ is irreducible.

Proof of Proposition 3.2. Let $\left\{N_{n}\right\}_{n \geq 0}$ be a defining sequence of $\Sigma \subset S^{3}$. Then we have $S^{3} \supset N=N_{0} \supset N_{1} \supset N_{2} \supset \cdots \supset N_{n} \supset \cdots \supset \Sigma=\cap_{n=1}^{\infty} N_{n}$ and

$$
N_{0} \backslash N_{1} \subset N_{0} \backslash N_{2} \subset \cdots \subset N_{0} \backslash N_{n} \subset \cdots \subset N_{0} \backslash \Sigma=\cup_{n=1}^{\infty} N_{0} \backslash N_{n} .
$$

Moreover, since the winding number of $N_{n+1}$ in $N_{n}$ is greater than 1 for every $n, \partial N_{n}$ is not parallel to $\partial N_{j}$ for $n \neq j$. 
Now we refine the defining sequence $\left\{N_{n}\right\}_{n \geq 0}$ to a maximal one. Let $\Gamma_{n}$ be the JSJ-decomposition tori of $N_{n} \backslash N_{n+1}$. Note that the set $\bigcup_{0 \leq n<j} \Gamma_{n} \cup \bigcup_{0<n<j} \partial N_{n}$ is the JSJ-decomposition tori of $N_{0} \backslash N_{j}$. Define $\Gamma(\Sigma)=\bigcup_{n>0}\left(\Gamma_{n} \cup \partial N_{n}\right)$. By Lemma 3.3 (1) (2) we can re-index the components of $\Gamma(\Sigma)$ as $\left\{T_{n}\right\}_{n \geq 0}$ so that

(1) each $T_{n}$ bounds a solid torus $N_{n}^{*}$,

(2) each $N_{n+1}^{*}$ is a thick braid in $N_{n}^{*}$,

(3) each $N_{n}^{*} \backslash N_{n+1}^{*}$ contains no essential torus.

Clearly $\left\{N_{n}\right\}_{n \geq 0}$ is a subsequence of $\left\{N_{n}^{*}\right\}_{n \geq 0}$ and by definition $\left\{N_{n}^{*}\right\}_{n \geq 0}$ is a maximal defining sequence of $\Sigma$.

Let $\left\{N_{n}^{\prime}\right\}_{n \geq 0}$ be another maximal defining sequence of $\Sigma \subset S^{3}$. We shall show that $\left\{N_{n}^{\prime}\right\}_{n \geq 0}$ and $\left\{N_{n}^{*}\right\}_{n \geq 0}$ are equivalent. Since $\Sigma$ is compact, we have $N_{j}^{*} \subset N_{k}^{\prime} \subset N_{0}^{*}$ for some big integers $j, k$. By Lemma 3.3 (3) and the construction of $\left\{N_{n}^{*}\right\}_{n \geq 0}, \partial N_{k}^{\prime}$ is isotopic in $N_{0}^{*} \backslash N_{j}^{*}$ to some $\partial N_{m}^{*}$. Clearly, the isotopy automatically sends $N_{k}^{\prime}$ to $N_{m}^{*}$.

Similarly, one argues that $N_{k+1}^{\prime}$ can be further isotoped in $N_{m}^{*}$ relative to $\Sigma$ to some $N_{m^{\prime}}^{*}$, in which $m^{\prime}$ must be $m+1$ because $N_{k}^{\prime} \backslash N_{k+1}^{\prime}$ contains no essential torus, and so on. Hence we verify that $\left\{N_{k+n}^{\prime}\right\}_{n \geq 0}$ is strongly equivalent to $\left\{N_{m+n}^{*}\right\}_{n \geq 0}$ and the conclusion follows.

\subsection{Classification of tame solenoids}

Theorem 3.4. Let $\Sigma, \Sigma^{\prime} \subset S^{3}$ be two tame solenoids. The following statements are equivalent.

(1) $\Sigma, \Sigma^{\prime}$ are equivalent.

(2) Some defining sequences of $\Sigma, \Sigma^{\prime}$ are equivalent.

(3) The maximal defining sequences of $\Sigma, \Sigma^{\prime}$ are equivalent.

Proof. $(2) \Rightarrow(1)$. Without loss of generality, suppose the defining sequences $\left\{N_{n}\right\}_{n \geq 0}$, $\left\{N_{n}^{\prime}\right\}_{n \geq 0}$ of $\Sigma, \Sigma^{\prime}$ are strongly equivalent. By definition there are orientation preserving homeomorphism $f_{0}:\left(S^{3}, N_{0}\right) \rightarrow\left(S^{3}, N_{0}^{\prime}\right)$ and orientation preserving homeomorphisms $f_{n}:\left(N_{n-1}, N_{n}\right) \rightarrow\left(N_{n-1}^{\prime}, N_{n}^{\prime}\right)$ with $\left.f_{n}\right|_{\partial N_{n-1}}=\left.f_{n-1}\right|_{\partial N_{n-1}}$ for $n \geq 1$.

By Remark 2.5, we assume the $D^{2}$-slices of all $N_{i}$, (respectively of all $N_{i}^{\prime}$ ), are coherent. Then it is easy to see that we can first isotopy $f_{0}:\left(S^{3}, N_{0}\right) \rightarrow\left(S^{3}, N_{0}^{\prime}\right)$ so that $f_{0} \mid: N_{0} \rightarrow N_{0}^{\prime}$ is $D^{2}$-fiberation preserving, then inductively to isotopy $f_{n}$ : $\left(N_{n-1}, N_{n}\right) \rightarrow\left(N_{n-1}^{\prime}, N_{n}^{\prime}\right)$ for each $n \geq 1$ so that $f_{n} \mid: N_{n} \rightarrow N_{n}^{\prime}$ is $D^{2}$-fiberation preserving, and still $\left.f_{n}\right|_{\partial N_{n-1}}=\left.f_{n-1}\right|_{\partial N_{n-1}}$.

To apply Lemma 2.9, we set $U_{n}=N_{n}$ and extend $f_{n}$ onto $S^{3}$ by setting $\left.f_{n}\right|_{S^{3} \backslash N_{n-1}}=$ $\left.f_{n-1}\right|_{S^{3} \backslash N_{n-1}}$. Clearly Conditions (1) and (3) of Lemma 2.9 are satisfied. Since the diameters of the meridian disks of $N_{n}$ and $N_{n}^{\prime}$ tend to zero uniformly as $n \rightarrow \infty$ and since $f_{n}$ is $D^{2}$-fiberation preserving and $\left.f_{n}\right|_{\partial N_{n-1}}=\left.f_{n-1}\right|_{\partial N_{n-1}}$, Condition (2) of Lemma 2.9 is also satisfied. Therefore, by Lemma $2.9 f_{n}$ uniformly converges to 
a homeomorphism $f:\left(S^{3}, \cap_{n \geq 0} N_{n}\right) \rightarrow\left(S^{3}, \cap_{n \geq 0} N_{n}^{\prime}\right)$. That is, $\Sigma=\cap_{n \geq 0} N_{n}$ and $\Sigma^{\prime}=\cap_{n \geq 0} N_{n}^{\prime}$ are equivalent.

$(3) \Rightarrow(2)$ is obvious.

$(1) \Rightarrow(3)$. Let $f: S^{3} \rightarrow S^{3}$ be an orientation preserving homeomorphism such that $f(\Sigma)=\Sigma^{\prime}$. Clearly for each maximal defining sequence $\left\{N_{n}\right\}_{n \geq 0}$ of $\Sigma,\left\{f\left(N_{n}\right)\right\}_{n \geq 0}$ is a maximal defining sequence of $\Sigma^{\prime}$ and is equivalent to $\left\{N_{n}\right\}_{n \geq 0}$. By Proposition 3.2. $\left\{f\left(N_{n}\right)\right\}_{n \geq 0}$, hence $\left\{N_{n}\right\}_{n \geq 0}$, is equivalent to every maximal defining sequence of $\Sigma^{\prime}$.

\subsection{Knotting, linking and invariants}

Thanks to the classification theorem, we can talk about the knotting, linking and invariants of tame solenoids.

Definition 3.5. Call a tame embedding of solenoid $\Sigma \subset S^{3}$ with defining sequence $\left\{N_{n}\right\}_{n \geq 0}$ is knotted, if some defining solid torus $N_{n} \subset S^{3}$ is knotted; otherwise call it unknotted.

Note that for a defining sequence $\left\{N_{n}\right\}_{n \geq 0}$ of a tame solenoid, if $N_{n}$ is knotted then so is $N_{n^{\prime}}$ for $n^{\prime}>n$. It follows from Theorem 3.4 that the notion of knotting is well defined for an equivalent class of tame solenoids.

Definition 3.6. Let $\Sigma, \Sigma^{\prime} \subset S^{3}$ be disjoint tame solenoids with disjoint defining sequences $\left\{N_{n}\right\}_{n \geq 0}$ and $\left\{N_{j}^{\prime}\right\}_{j \geq 0}$ respectively.

(1) Call $\Sigma, \Sigma^{\prime}$ algebraically linked if some linking number $l k\left(N_{n}, N_{j}^{\prime}\right)$ (i.e. the linking number of their centerlines) is non-zero.

(2) Call $\Sigma, \Sigma^{\prime}$ linked if some defining solid tori $N_{n}, N_{j}^{\prime}$ are linked.

Since two disjoint tame solenoids $\Sigma, \Sigma^{\prime} \subset S^{3}$ always have disjoint defining sequences and since $l k\left(N_{n}, N_{j}^{\prime}\right) \neq 0$ implies $l k\left(N_{n^{\prime}}, N_{j^{\prime}}^{\prime}\right) \neq 0$ for all $n^{\prime} \geq n, j^{\prime} \geq j$, by Theorem 3.4 again the notion of algebraic linking is well defined for equivalent classes of tame solenoids.

Similarly the notion of linking is well defined, too. In particular, $\Sigma$ and $\Sigma^{\prime}$ are linked if and only if there are no disjoint 3-balls $B$ and $B^{\prime}$ such that $\Sigma \subset B, \Sigma^{\prime} \subset B^{\prime}$.

To define invariants of tame solenoids, the proposition below will be of help.

Proposition 3.7. Up to strong equivalence, each knotted tame solenoid $\Sigma \subset S^{3}$ has a unique maximal defining sequence $\left\{N_{n}\right\}_{n \geq 0}$ such that $N_{0}$ is knotted and any other defining sequence $\left\{N_{n}^{\prime}\right\}_{n \geq 0}$ with knotted $N_{0}^{\prime}$ is a subsequence of $\left\{N_{n}\right\}_{n \geq 0}$.

Proof. Follow the proof of Proposition 3.2. Assume $N_{0}$ is knotted and append further into $\Gamma(\Sigma)$ all such JSJ-decomposition torus of $S^{3} \backslash N_{0}$ that bounds a solid tours in $S^{3}$, in which $N_{0}$ is a thick braid. It is a routine matter to verify that the resulting maximal defining sequence is exactly what we want. 
For any knot invariant I (for example, the genus, the Gromov volume, the Alexander polynomial or the Jones polynomial) one has an invariant $I$ of tame solenoids as below. For a knotted tame solenoid $\Sigma \subset S^{3}$, let $\left\{N_{n}\right\}_{n \geq 0}$ be the unique maximal defining sequence from the above proposition. Then the infinite sequence $I(\Sigma)=$ $\left\{I\left(N_{0}\right), I\left(N_{1}\right), \ldots, I\left(N_{n}\right), \ldots\right\}$ depends only on the equivalence class of $\Sigma$. If a tame solenoid $\Sigma \subset S^{3}$ is unknotted, then for any defining sequence $\left\{N_{n}\right\}_{n \geq 0}$ of $\Sigma$ the sequence $I(\Sigma)=\left\{I\left(N_{0}\right), I\left(N_{1}\right), \ldots, I\left(N_{n}\right), \ldots\right\}$ is identically trivial, say $\{0,0, \ldots, 0, \ldots\}$, if $I$ is either the genus or the Gromov volume, or $\{1,1, \ldots, 1, \ldots\}$, if $I$ is either the Alexander polynomial or the Jones polynomial.

In general for given numerical function $g$ and knot invariant $I$, one may organize the sequence $I(\Sigma)$ into a formal series $I(\Sigma, g)=\sum_{n=0}^{\infty} g(n) I\left(N_{n}\right) t^{n}$. We wonder if $I(\Sigma, g)$ would have interesting properties for certain $g$ and $I$ as well as for suitable classes of solenoids.

\subsection{Unknotted 2-adic tame solenoids}

Given an unknotted solid torus $N$ in $S^{3}$, there are exactly two kinds of thick braids of winding number two in $N$ that are unknotted in $S^{3}$ as shown in Figure 2, where the left one is the left-handed embedding, which we denote by -1 , and the right one is the right-handed embedding, which we denote by +1 . Then any maximal defining sequence of a unknotted 2-adic tame solenoids in $S^{3}$ can be presented as an infinite sequence of \pm 1 .

Let $Z_{2}$ be the set of infinite sequences $\left(a_{1}, a_{2}, \ldots, a_{n}, \ldots\right)$ of \pm 1 . Two such sequences are said to be equivalent, if they can be made identical by deleting finitely many terms. By Theorem 3.4 the equivalence classes of unknotted 2-adic tame solenoids are in 1-1 correspondence to the equivalence classes of $Z_{2}$. In particular, there are uncountably many equivalence classes of unknotted 2-adic tame solenoids.
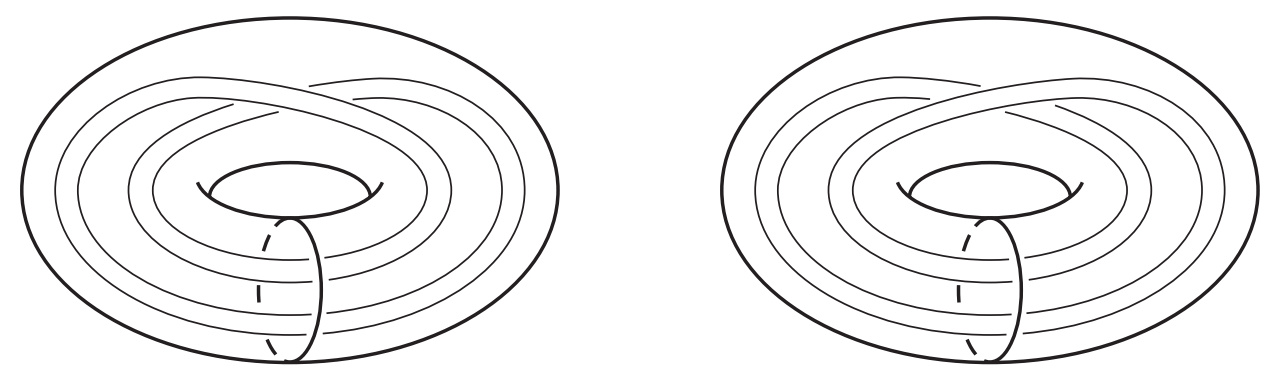

Figure 2 


\subsection{Smale solenoids}

The solenoids were introduced into dynamics by Smale as hyperbolic attractors in [S].

Definition 3.8. Let $M$ be a 3-manifold and $f: M \rightarrow M$ be a homeomorphism. If there is a solid torus $N \subset M$ such that $\left.f\right|_{N}$ (resp. $\left.f^{-1}\right|_{N}$ ) defines a thick braid as in Definition 2.2 (1), we call the hyperbolic attractor $\Sigma=\cap_{n=1}^{\infty} f^{n}(N)$ (resp. the hyperbolic repeller $\left.\Sigma=\cap_{n=1}^{\infty} f^{-n}(N)\right)$ a Smale solenoid.

Clearly each Smale solenoid $\Sigma \subset S^{3}$ is tame. It is known that a Smale solenoid $\Sigma \subset S^{3}$ must be unknotted [JNW]. Moreover, it is proved in [JNW] that if the nonwondering set $w(f)$ of a dynamics $f$ consists of finitely many disjoint Smale solenoids, then $w(f)$ consists of two solenoids (indeed they are algebraically linked).

Definition 3.9. Let $w_{1}, \ldots, w_{k}$ be integers greater than 1. Call a Smale solenoid $\Sigma \subset S^{3}$ is of type $\left(w_{1}, \ldots, w_{k}\right)$, if $(1)$ there is a dynamics $f$ taking $\Sigma$ as an attractor, (2) there is a defining sequence $\left\{N_{n}\right\}_{n \geq 0}$ of $\Sigma$ such that $f$ sends $N_{n}$ to $N_{k+n}$ for all $n \geq 0$ and (3) $w_{n}$ is the winding number of $N_{n}$ in $N_{n-1}$ for $1 \leq n \leq k$.

Proposition 3.10. Any given type $\left(w_{1}, \ldots, w_{k}\right)$ is realized by a Smale solenoid $\Sigma \subset S^{3}$. Moreover, the number of Smale solenoids $\Sigma \subset S^{3}$ of type $\left(w_{1}, \ldots, w_{k}\right)$ is finite if all $w_{n} \leq 3$, and is countably infinite otherwise.

Proof. First, we extend the sequence $\left(w_{1}, \ldots, w_{k}\right)$ to a infinite one by setting $w_{k+n}=$ $w_{n}$. Then choosing an unknotted solid tours $N_{0} \subset S^{3}$ and letting $N_{n} \subset N_{n-1}$ be a tubular neighborhood of $\overline{\sigma_{1} \cdots \sigma_{w_{n}-1}}$ give rise to a defining sequence of the desired Smale solenoid, where $\sigma_{i}$ 's are standard generators of the braid groups.

The "Moreover" part follows from the lemma below and Theorem 3.4.

Lemma $3.11([\mathrm{MP}])$. Let $W_{n}$ be the set of $n$-strand braids whose closures are unknotted in $S^{3}$. Then

(1) $W_{n}$ has two conjugacy classes as pictured in Figure 2 for $n=2$;

(2) $W_{n}$ has three conjugacy classes as pictured in Figure 3 for $n=3$;

(3) $W_{n}$ has infinitely many conjugacy classes for $n>3$.
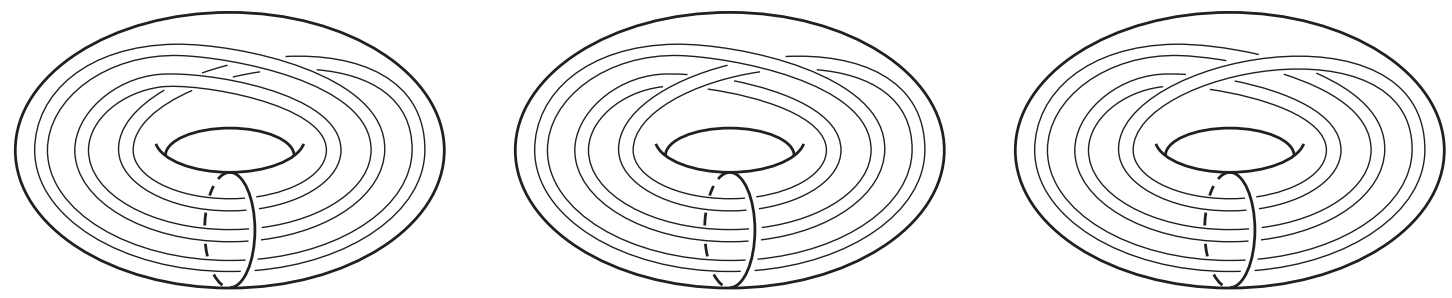

Figure 3 


\section{Chirality of tame solenoids}

Definition 4.1. Call a subset $A \subset S^{3}$ achiral, if there is an orientation reversing homeomorphism $r: S^{3} \rightarrow S^{3}$ such that $r(A)=A$. Call $A$ strictly achiral, if there is an orientation reversing homeomorphism $r: S^{3} \rightarrow S^{3}$ such that $r(x)=x$ for every $x \in A$.

In Definition 4.1, "achiral" means setwise achiral, and "strictly achiral" means pointwise achiral. They are two oppiste extremes among various shades of achirality in the real word.

\subsection{Criterions}

By definition, a tame solenoid in $S^{3}$ is achiral if and only if it is equivalent to its mirror image. Therefore, by Theorem 3.4 we have the following criterion of the chirality of tame solenoids.

Theorem 4.2. A tame solenoid given by the maximal defining sequence $\left\{N_{n}\right\}_{n \geq 0}$ is achiral if and only if $\left\{N_{n}\right\}_{n \geq 0}$ is equivalent to its mirror image.

Example 4.3. Recall the example in $§ 3.4$. The mirror image of a maximal defining sequence of a unknotted 2-adic tame solenoid presented by $\left(a_{1}, a_{2}, \ldots, a_{n}, \ldots\right)$ is presented by $\left(-a_{1},-a_{2}, \ldots,-a_{n}, \ldots\right)$. Therefore, by Theorem 4.2, the unknotted 2adic tame solenoid presented by $(+1,-1,+1,-1, \ldots)$ is achiral but those solenoids presented by $(+1,+1,+1,+1, \ldots)$ or $(-1,-1,-1,-1, \ldots)$ are not achiral.

Below we focus on the strict achirality of tame solenoids. For a map $f: X \rightarrow X$, we use $\operatorname{Fix}(f)$ to denote the fixed point set of $f$.

Definition 4.4. Suppose $A$ is a subset of the solid torus $N$ and $l$ is a given framing of $N$. Call $A$ is strictly achiral with respect to $l$ if there exists an orientation reversing homeomorphism $f: N \rightarrow N$ such that $A \cup l \subset \operatorname{Fix}(f)$.

Remark 4.5. It is well known that the orientation reversing homeomorphism of $S^{3}$ is unique up to isotopy, but this is not true for the solid tours. The ambiguity can be removed by posing the framing fixing condition used in the above definition.

To prove the main theorem of this subsection, we need the following two lemmas.

Lemma 4.6. Suppose $l^{\prime}$ is a framing of a thick braid in $N$. If $l^{\prime}$ is strictly achiral with respect to a framing $l$ of $N$, then the strict achirality can be given by a $D^{2}$-fiberation preserving homeomorphism of $N$. 
Proof. Suppose the strict achirality of $l^{\prime} \subset N$ with respect to $l$ is defined by an orientation reversing homeomorphism $r: N \rightarrow N$. Then we can isotope $r$ relative to $l^{\prime} \cup l$ to a $D^{2}$-fiberation preserving homeomorphism $r^{\prime}$ : since we can slide $r\left(D^{2} \times *\right)$ relative to $l^{\prime} \cup l$ to $D^{2} \times *$, and the isotopy is just the process of this sliding. (More clear way to see this sliding: let $p: \tilde{N} \rightarrow N$ be the infinite cyclic covering, and $\tilde{l}^{\prime} \subset \tilde{N}$ be the preimage of $l^{\prime}$, then $\left(\tilde{N}, \tilde{l}^{\prime}\right)$ is homeomorphic to $\left(D^{2}, w\right.$ points $) \times \mathbb{R}$ where $w$ is the winding number of $l^{\prime}$ in $N$.)

Lemma 4.7. Suppose $\Sigma \subset S^{3}$ is a tame embedding given by a defining sequence $\left\{N_{n}\right\}_{n \geq 0}$. If $\Sigma \subset \operatorname{Fix}(r)$ for some homeomorphism $r: S^{3} \rightarrow S^{3}$, then there exists $k>0$ such that $r\left(N_{n}\right) \subset$ int $N_{0}$, and moreover $r\left(N_{n}\right), N_{n}$ have the same winding number in $N_{0}$ for $n \geq k$.

Proof. Since $S^{3}$ is compact, $r$ is uniformly continuous.

(i) Let $\epsilon=d\left(N_{1}, \partial N_{0}\right) / 2$.

(ii) Choose $0<\delta<\epsilon$ such that if $d\left(x, x^{\prime}\right)<\delta$ then $d\left(r(x), r\left(x^{\prime}\right)\right)<\epsilon$.

(iii) Choose $k>0$ such that $\max _{x \in N_{k}} d(x, \Sigma)<\delta$.

Now fix an integer $n \geq k$. For any $x \in N_{n}$, by (i) we have $d\left(x, \partial N_{0}\right) \geq 2 \epsilon$ and by (iii) we can choose $x^{\prime} \in \Sigma$ such that $d\left(x, x^{\prime}\right)<\delta$, hence by (ii) we have

$$
d(x, r(x)) \leq d\left(x, x^{\prime}\right)+d\left(x^{\prime}, r\left(x^{\prime}\right)\right)+d\left(r\left(x^{\prime}\right), r(x)\right)<\delta+0+\epsilon<2 \epsilon \leq d\left(x, \partial N_{0}\right) .
$$

It follows that the unique geodesic $\alpha(x)$ connecting $x$ and $r(x)$ lies in int $N_{0}$. Therefore, $\left\{\alpha(x) \mid x \in N_{n}\right\}$ gives rise to a homotopy from $N_{n}$ to $r\left(N_{n}\right)$ in $N_{0}$. In particular, $r\left(N_{n}\right) \subset \operatorname{int} N_{0}$ and $r\left(N_{n}\right), N_{n}$ have the same winding number in $N_{0}$.

Theorem 4.8. Let $\Sigma \subset S^{3}$ be a tame solenoid with defining sequence $\left\{N_{n}\right\}_{n \geq 0}$ and let $l_{n}$ denote a zero framing of $N_{n}$ in $S^{3}$, that is, $l_{n}$ is null-homologous in $S^{3} \backslash N_{n}$. Then $\Sigma$ is strictly achiral if and only if there exists $k \geq 0$ such that the $l_{k}$ is strictly achiral in $S^{3}$, and $l_{n+1}$ is strictly achiral in $N_{n}$ with respect to $l_{n}$ for all $n \geq k$.

Proof. Sufficiency. Without loss of generality, we assume $k=0$. Since $l_{0}$ is strictly achiral, there is an orientation reversing homeomorphism $f_{0}:\left(S^{3}, N_{0}\right) \rightarrow\left(S^{3}, N_{0}\right)$ such that $\left.f_{0}\right|_{N_{0}}$ is $D^{2}$-fiberation preserving and fixes $l_{0}$ pointwisely.

Since $l_{1}$ is strictly achiral in $N_{0}$ with respect to $l_{0}$, by Lemma 4.6 there is an orientation reversing and $D^{2}$-fiberation preserving homeomorphism $f_{1}:\left(N_{0}, N_{1}\right) \rightarrow\left(N_{0}, N_{1}\right)$ such that $l_{1} \cup l_{0}$ stays in $\operatorname{Fix}\left(f_{1}\right)$. Since both $\left.f_{0}\right|_{N_{0}}$ and $f_{1}$ are orientation reversing and $D^{2}$-fiberation preserving homeomorphisms of $N_{0}$ and both fix $l_{0}$ pointwisely, we may assume $\left.f_{1}\right|_{\partial N_{0}}=\left.f_{0}\right|_{\partial N_{0}}$. So $f_{1}$ may be extended onto $S^{3}$ by setting $\left.f_{1}\right|_{S^{3} \backslash N_{0}}=\left.f_{0}\right|_{S^{3} \backslash N_{0}}$.

Then for $n>1$, by the same reason we can recursively define homeomorphism $f_{n}:\left(S^{3}, N_{n}\right) \rightarrow\left(S^{3}, N_{n}\right)$ such that $\left.f_{n}\right|_{S^{3} \backslash N_{n-1}}=\left.f_{n-1}\right|_{S^{3} \backslash N_{n-1}}$ and that $\left.f_{n}\right|_{N_{n-1}}$ is $D^{2}$-fiberation preserving and fixes $l_{n} \cup l_{n-1}$ pointwisely.

To apply Lemma 2.9, set $U_{n}=N_{n}$. Clearly Conditions (1) and (3) of Lemma 2.9 are satisfied. Since $\left.f_{n}\right|_{N_{n}}$ is $D^{2}$-fiberation preserving and the diameters of the meridian 
disks of $N_{n}$ tend to zero uniformly as $n \rightarrow \infty$, Conditions (2) and (4) of Lemma 2.9 are also satisfied. By Lemma 2.9, $f_{n}$ uniformly converges to an orientation reversing homeomorphism $f: S^{3} \rightarrow S^{3}$ with the property that $\cap_{n \geq 0} N_{n} \subset \operatorname{Fix}(f)$.

Necessity. Suppose the strictly achirality of $\Sigma$ is defined by an orientation reversing homeomorphism $r$ and let $k>0$ be given by Lemma 4.7. Then for any $n \geq k$ we have that both $N_{n}$ and $r\left(N_{n}\right)$ are contained in the interior of $N_{0}$.

Fix an integer $n \geq k$ and choose a big integer $j$ so that $N_{j} \subset N_{n} \cap r\left(N_{n}\right)$. By Lemma 3.3 (3), both $\partial N_{n}, r\left(\partial N_{n}\right)$ are isotopic in $N_{0} \backslash N_{j}$ to some components of $\Gamma \cup \partial N_{0} \cup \partial N_{j}$ where $\Gamma$ is the JSJ-decomposition tori of $N_{0} \backslash N_{j}$. Therefore, we can isotope $r$ with support in $N_{0} \backslash \Sigma$ so that either $r\left(N_{n}\right)=N_{n}$, or by Lemma 3.3 (1) $r\left(N_{n}\right) \subset N_{n}$ or $N_{n} \subset r\left(N_{n}\right)$ is a thick braid of winding number greater than 1. By Lemma 4.7 $r\left(N_{n}\right), N_{n}$ have the same winding number in $N_{0}$, so the latter case cannot happen and, moreover, we may assume the zero framing $l_{n}$ of $N_{n}$ lies in $\operatorname{Fix}(r)$.

By the same argument, $r$ can be further isotoped with support in $N_{n} \backslash \Sigma$ so that $r\left(N_{n+1}\right)=N_{n+1}$ and $l_{n+1} \subset \operatorname{Fix}(r)$. Therefore, $l_{n}$ is strictly achiral in $S^{3}$ and $l_{n+1}$ is strictly achiral in $N_{n}$ with respect to $l_{n}$.

\subsection{Examples}

Thanks to Theorem 4.8, the strict achirality of tame solenoids is the problem of strict achirality of knots in $S^{3}$ and closed braids in the solid torus. Below we fix a point $* \in \partial D^{2}$ and let $l_{*}$ denote the framing $* \times S^{1}$ of $D^{2} \times S^{1}$.

Definition 4.9. Call a braid $\beta$ achiral, if $\beta$ is conjugate to its mirror image $\beta^{*}$.

Note that the achirality is well defined for a conjugacy class of braids. Also note that the closure $\bar{\beta} \subset D^{2} \times S^{1}$ of a braid $\beta$ is connected if and only if $\beta$ is cyclic, i.e. $\beta$ permutes its strands cyclicly.

Proposition 4.10. For every cyclic braid $\beta$, the closure $\bar{\beta} \subset D^{2} \times S^{1}$ is strictly achiral with respect to $l_{*}$ if and only if $\beta$ is achiral.

Proof. Sufficiency. Suppose $\beta=\alpha^{-1} \beta^{*} \alpha$. Then $\beta=\left(\alpha \beta^{k}\right)^{-1} \beta^{*}\left(\alpha \beta^{k}\right)$ for any integer $k$. Since $\beta$ and $\beta^{*}$ give rise to the same cyclic permutation on their strands, it follows from the equality $\beta=\alpha^{-1} \beta^{*} \alpha$ that the permutation given by $\alpha$ is commutative with hence is a power of that given by $\beta$. So we can choose $k$ so that $\alpha \beta^{k}$ is a pure braid. Replacing $\alpha$ by $\alpha \beta^{k}$, we may assume $\alpha$ is a pure braid.

By the definition of braid, there is a $D^{2}$-fiberation preserving and boundary fixing homeomorphism $\tilde{g}: D^{2} \times[0,1] \rightarrow D^{2} \times[0,1]$ which sends $\alpha^{-1} \beta^{*} \alpha$ to $\beta$. Then $\tilde{g}$ induces a homeomorphism $g: D^{2} \times S^{1} \rightarrow D^{2} \times S^{1}$ which sends $\overline{\alpha^{-1} \beta^{*} \alpha}$ to $\bar{\beta}$. Moreover, by canceling the $\alpha$ part and the $\alpha^{-1}$ part of $\overline{\alpha^{-1} \beta^{*} \alpha}$, we can define a $D^{2}$-fiberation preserving and boundary fixing homeomorphism $f: D^{2} \times S^{1} \rightarrow D^{2} \times S^{1}$ which sends $\overline{\beta^{*}}$ to $\overline{\alpha^{-1} \beta^{*} \alpha}$. 
Finally, let $r: D^{2} \times S^{1} \rightarrow D^{2} \times S^{1}$ be the reflection about the "page" (assume $l_{*}$ lies on it). Then $g f r: D^{2} \times S^{1} \rightarrow D^{2} \times S^{1}$ is an orientation reversing homeomorphism and sends $\bar{\beta}$ to $\bar{\beta}$. Since $\alpha$ is a pure braid and all $g, f, r$ preserve each $D^{2}$ slice of $D^{2} \times S^{1}$, it follows that $g f r$ fixes both the closure $\bar{\beta}$ and the framing $l_{*}$ pointwisely.

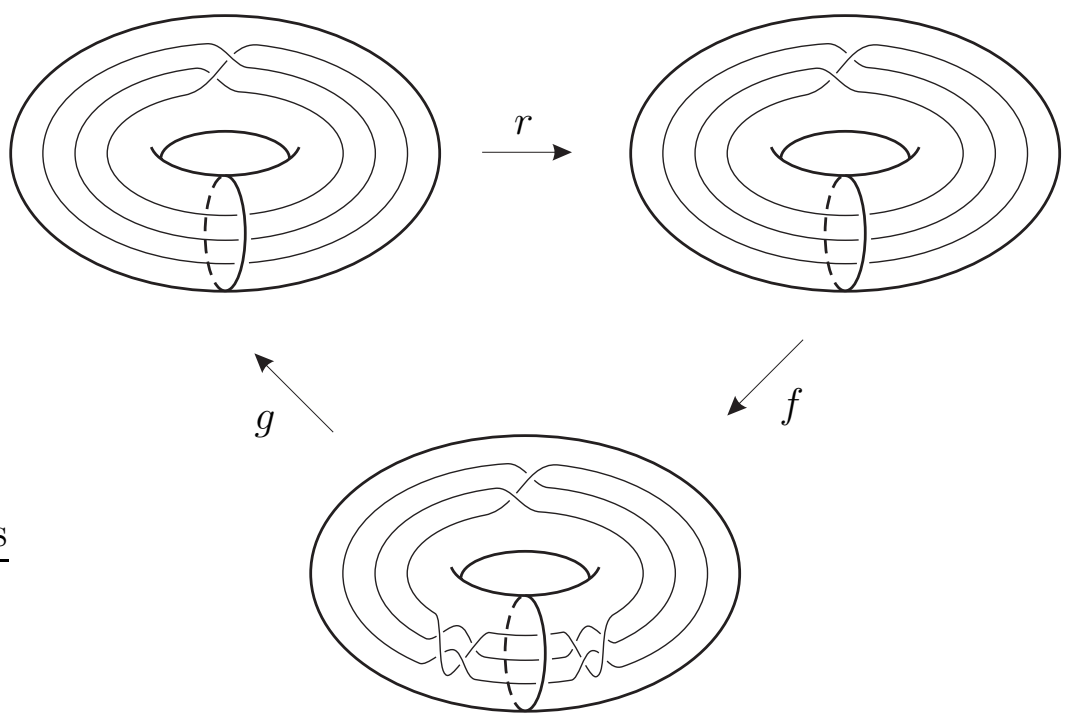

Figure 4

Figure 4 illustrates the case $\beta=\sigma_{1} \sigma_{2}^{-1}$ and $\alpha=\sigma_{2} \sigma_{1}^{2} \sigma_{2}^{-1}$ (see Example 4.11 for the equality $\beta=\alpha^{-1} \beta^{*} \alpha$ ).

Necessity. If $\bar{\beta}$ is strictly achiral with respect to $l_{*}$ then $\bar{\beta}$ is isotopic to its mirror image, therefore $\beta$ is conjugate to its mirror image $\beta^{*}$.

Example 4.11. Examples of cyclic, achiral braids.

(1) $\beta=\sigma_{1} \sigma_{2}^{-1}$ is cyclic and achiral. Setting $\alpha=\sigma_{2} \sigma_{1}^{2} \sigma_{2}^{-1}$, one can verify the equality $\beta=\alpha^{-1} \beta^{*} \alpha$ either by directly a braid move or by the substitution of braid relation $\sigma_{2} \sigma_{1} \sigma_{2}=\sigma_{1} \sigma_{2} \sigma_{1}$ as follows

$$
\begin{aligned}
\alpha^{-1} \beta^{*} \alpha & =\left(\sigma_{2} \sigma_{1}^{-1} \sigma_{1}^{-1} \sigma_{2}^{-1}\right)\left(\sigma_{1}^{-1} \sigma_{2}\right)\left(\sigma_{2} \sigma_{1} \sigma_{1} \sigma_{2}^{-1}\right) \\
& =\sigma_{2} \sigma_{1}^{-1} \sigma_{2}^{-1} \sigma_{1}^{-1} \sigma_{2}^{-1} \sigma_{2} \sigma_{2} \sigma_{1} \sigma_{1} \sigma_{2}^{-1} \\
& =\sigma_{2} \sigma_{2}^{-1} \sigma_{1}^{-1} \sigma_{2}^{-1} \sigma_{2}^{-1} \sigma_{2} \sigma_{2} \sigma_{1} \sigma_{1} \sigma_{2}^{-1} \\
& =\sigma_{1} \sigma_{2}^{-1}=\beta .
\end{aligned}
$$

(2) For any braid $\beta, \beta \beta^{*}$ is achiral, since

$$
\beta^{*-1}\left(\beta \beta^{*}\right)^{*} \beta^{*}=\beta^{*-1} \beta^{*} \beta \beta^{*}=\beta \beta^{*} .
$$

Moreover, for any cyclic braid $\beta$ of odd number of strands, $\beta \beta^{*}$ is also cyclic. Hence for each cyclic braid $\beta$ of odd number of strands, $\beta \beta^{*}$ is cyclic and achiral. 
(3) If $\beta$ is an achiral braid, then so is $\beta^{k}$ for any integer $k$. Moreover, if $\beta$ is cyclic, then so is $\beta^{k}$ for every integer $k$ relatively prime to the number of strands.

Proposition 4.12. (1) If a connected closed braid $\bar{\beta} \subset D^{2} \times S^{1}$ is strictly achiral with respect to $l_{*}$, then the writhe of $\bar{\beta}$ is zero (for the definition of writhe, see [A,p.152]).

(2) Hence a connected closed braid $\bar{\beta} \subset D^{2} \times S^{1}$ is not strictly achiral with respect to $l_{*}$ if $\bar{\beta}$ is either of even winding number, or a cable.

Proof. (1) By Proposition 4.10, $\bar{\beta}$ is strictly achiral implies $\beta=\alpha^{-1} \beta^{*} \alpha$ for some braid $\alpha$. Clearly $w r(\bar{\beta})=w r\left(\overline{\alpha^{-1} \beta^{*} \alpha}\right)=w r\left(\overline{\beta^{*}}\right)=-w r(\bar{\beta})$. It follows that $w r(\bar{\beta})=0$.

(2) Suppose $\bar{\beta}$ is connected and is of even winding number. It is an elementary exercise to show that the number of the crossings of $\bar{\beta}$ is odd. Hence $w r(\bar{\beta})$ must be odd, which contradicts (1).

Suppose $\bar{\beta}$ is a cable. Then all the crossings of $\bar{\beta}$ have the same sign, so $w r(\bar{\beta})$ is non-zero, which contradicts (1).

Now we state the main result of this subsection.

Theorem 4.13. A solenoid of type $\varpi=\left(w_{1}, w_{2}, \ldots, w_{n}, \ldots\right)$ has a strictly achiral tame embedding into $S^{3}$ if and only if all except finitely many $w_{n}$ are odd.

Proof. Necessity is immediate from Theorem 4.8 and Proposition 4.12 (2).

Sufficiency. Assume all $w_{n}$ are odd. Let $N_{0}$ be a tubular neighborhood of a strictly achiral knot in $S^{3}$ and let $N_{n}$ be a tubular neighborhood of $\overline{\beta_{n} \beta_{n}^{*}}$ in $N_{n-1}$ where $\beta_{n}$ is an arbitrary cyclic braid on $w_{n}$ strands. By Theorem 4.8, Proposition 4.10 and Example 4.11 (2) the defining sequence $\left\{N_{n}\right\}_{n \geq 0}$ gives rise to a strictly achiral tame embedding of the solenoid of type $\varpi=\left(w_{1}, w_{2}, \ldots, w_{n}, \ldots\right)$.

Example 4.14. (1) The 2-adic solenoid has no strictly achiral tame embedding into $S^{3}$.

(2) By Lemma 3.11 (2), Example 4.11 (1) and Proposition 4.12 (2), up to equivalence the 3-adic solenoid has a unique unknotted strictly achiral tame embedding into $S^{3}$, which is yielded by nesting the thick braid pictured in the middle of Figure 3 .

(3) The embedding in Theorem 4.13 can be chosen to be either knotted or unknotted, by letting in the proof either $N_{0}$ be a tubular neighborhood of the figure- 8 knot, or letting $N_{0}$ be a tubular neighborhood of the unknot and $\beta_{n}=\sigma_{1} \sigma_{2} \cdots \sigma_{w_{n}-1}$ (we leave it to the reader to verify that the closure $\overline{\beta_{n} \beta_{n}^{*}}$ is unknotted in $S^{3}$ ).

\section{References}

[A] Adams, C. C. The knot book. An elementary introduction to the mathematical theory of knots, W. H. Freeman and Company, New York, 1994. 
[Bin] Bing, R. H. A simple closed curve is the only homogeneous bounded plane continuum that contains an arc, Canad. J. Math. 12 (1960), 209-230.

[Bir] Birman, J. S. Braids, links, and mapping class groups, Annals of Mathematics Studies, No. 82. Princeton University Press, Princeton, NJ, 1974.

[Ja] Jaco, W. H. Lectures on three-manifold topology, Regional Conference Series in Mathematics 43, Amer. Math. Soc., Providence, RI, 1980.

[JNW] Jiang, B.; Ni, Y.; Wang, S. 3-manifolds that admit knotted solenoids as attractors, Trans. Amer. Math. Soc. 356 (2004), no. 11, 4371-4382.

[JW] Jiang, B.; Wang, S. Achirality and planarity. Commun. Contemp. Math. 2 (2000), no. 3, 299-305.

[JWZ] Jiang, B.; Wang, S.; Zheng, H. No embedding of solenoids into surfaces, math.GT/0610820.

[MP] Magnus, W.; Peluso, A. On knot groups, Comm. Pure Appl. Math. 20 (1967), 749-770.

[Mc] McCord, M. C. Inverse limit sequences with covering maps, Trans. Amer. Math. Soc. 114 (1965), no. 1, 197-209.

[R] Robinson, C. Dynamical systems. Stability, symbolic dynamics, and chaos. Second edition. Studies in Advanced Mathematics, CRC Press, Boca Raton, FL, 1999.

[S] Smale, S. Differentiable dynamical systems, Bull. Amer. Math. Soc. 73 (1967), 747-817.

[V] Vietoris, L. Über den höheren Zusammenhang kompakter Räume und eine Klasse von zusammenhangstreuen Abbildungen, (German) Math. Ann. 97 (1927), no. $1,454-472$. 